\title{
In vivo Imaging of Cannabinoid Type 2 Receptors: Functional and Structural Alterations in Mouse Model of Cerebral Ischemia by PET and MRI
}

\author{
Ruiqing $\mathrm{Ni}^{1,2}{ }^{\circ}$, Adrienne Müller Herde ${ }^{3}$, Ahmed Haider ${ }^{3}$, Claudia Keller ${ }^{3}$, Georgios Louloudis ${ }^{1}$, \\ Markus Vaas ${ }^{1}$, Roger Schibli ${ }^{3}$, Simon M. Ametamey ${ }^{3}$, Jan Klohs ${ }^{1}$, and Linjing $\mathrm{Mu}^{3,4} \odot$ \\ ${ }^{1}$ Institute for Biomedical Engineering, University of Zurich \& ETH Zurich, Zurich, Switzerland \\ ${ }^{2}$ Institute for Regenerative Medicine, University of Zurich, Zurich, Switzerland \\ ${ }^{3}$ Department of Chemistry and Applied Biosciences, ETH Zurich, HCI H427 Vladimir-Prelog-Weg 1-5/10, 8093 Zurich, Switzerland \\ ${ }^{4}$ Department of Nuclear Medicine, University Hospital Zurich, Zurich, Switzerland 2021
}

\begin{abstract}
Purpose: Stroke is one of the most prevalent vascular diseases. Non-invasive molecular imaging methods have the potential to provide critical insights into the temporal dynamics and follow alterations of receptor expression and metabolism in ischemic stroke. The aim of this study was to assess the cannabinoid type 2 receptor $\left(\mathrm{CB}_{2} \mathrm{R}\right)$ levels in transient middle cerebral artery occlusion (tMCAO) mouse models at subacute stage using positron emission tomography (PET) with our novel tracer $\left[{ }^{18} \mathrm{~F}\right]$ RoSMA-18-d6 and structural imaging by magnetic resonance imaging (MRI).

Procedures: Our recently developed $\mathrm{CB}_{2} \mathrm{R}$ PET tracer $\left[{ }^{18} \mathrm{~F}\right] \mathrm{RoSMA}-18-\mathrm{d} 6$ was used for imaging neuroinflammation at $24 \mathrm{~h}$ after reperfusion in tMCAO mice. The RNA expression levels of $\mathrm{CB}_{2} \mathrm{R}$ and other inflammatory markers were analyzed by quantitative real-time polymerase chain reaction using brain tissues from tMCAO ( 1 h occlusion) and sham-operated mice. $\left[{ }^{18} \mathrm{~F}\right]$ fluorodeoxyglucose (FDG) was included for evaluation of the cerebral metabolic rate of glucose (CMRglc). In addition, diffusionweighted imaging and $\mathrm{T}_{2}$-weighted imaging were performed for anatomical reference and delineating the lesion in $\mathrm{MCAO}$ mice.

Results: mRNA expressions of inflammatory markers TNF- $a, l b a 1, M M P 9$ and GFAP, CNR2 were increased to $1.3-2.5$ fold at $24 \mathrm{~h}$ after reperfusion in the ipsilateral compared to contralateral hemisphere of tMCAO mice, while mRNA expression of the neuronal marker MAP-2 was markedly reduced to ca. $50 \%$. Reduced $\left[{ }^{18} \mathrm{~F}\right] F D G$ uptake was observed in the ischemic striatum of $\mathrm{tMCAO}$ mouse brain at $24 \mathrm{~h}$ after reperfusion. Although higher activity of $\left[{ }^{18} \mathrm{~F}\right]$ RoSMA-18-d6 in ex vivo biodistribution studies and higher standard uptake value ratio (SUVR) were detected in the ischemic ipsilateral compared to contralateral striatum in tMCAO mice, the in vivo specificity of $\left[{ }^{18} \mathrm{~F}\right] \mathrm{RoSMA}-18-\mathrm{d} 6$ was confirmed only in the $\mathrm{CB}_{2} \mathrm{R}$-rich spleen.

Conclusions: This study revealed an increased $\left[{ }^{18} \mathrm{~F}\right]$ RoSMA-18-d6 measure of $\mathrm{CB}_{2} \mathrm{R}$ and a reduced $\left[{ }^{18} \mathrm{~F}\right]$ FDG measure of CMRglc in the ischemic striatum of tMCAO mice at subacute stage. $\left[{ }^{18} \mathrm{~F}\right]$ RoSMA-18-d6 might be a promising $\mathrm{PET}$ tracer for detecting $\mathrm{CB}_{2} \mathrm{R}$ alterations in animal models of neuroinflammation without neuronal loss.
\end{abstract}

Key words Cannabinoid type 2 receptor $\cdot\left[{ }^{18} \mathrm{~F}\right]$ RoSMA-18-d6 $\cdot$ Ischemic stroke . Neuroinflammation · Magnetic resonance imaging $\cdot$ Positron emission tomography

Correspondence to: Linjing Mu; e-mail: linjing.mu@

pharma.ethz.ch 


\section{Introduction}

The pathophysiology of ischemic stroke is complex and associated with a myriad of cellular and molecular pathways. The severe reduction in cerebral blood flow (CBF) initiates a cascade of hemodynamic, vascular and inflammatory processes in a time-dependent manner in the supplied brain territory, and subsequent defensive response for repair related to lesion expansion and containment. Irreversible tissue damage occurs in the core of the ischemic area, while neurons in the ischemic penumbra face excitotoxicity, peri-infarct polarizations, inflammation, and apoptosis, leading to a secondary tissue damage and expansion of the lesion if reperfusion cannot be restored within an early time frame [1-3]. Neuroinflammation post stroke has been an important therapeutic target. Anti-inflammatory, immunomodulatory treatments and microglia-targeted therapy were evaluated in clinical stroke trials [4-6]. Thus, there is a need for imaging the regional neuroinflammatory pattern for understanding disease mechanism and for therapeutic monitoring.

Positron emission tomography (PET) using $\left[{ }^{18} \mathrm{~F}\right]$ fluorodeoxyglucose $\left(\left[{ }^{18} \mathrm{~F}\right] \mathrm{FDG}\right)$ for cerebral metabolic rate of glucose (CMRglc), $\left[{ }^{15} \mathrm{O}\right] \mathrm{H}_{2} \mathrm{O}$ for perfusion imaging, and diffusion weighted (DW) magnetic resonance imaging (MRI) are valuable tools to support understanding of the pathophysiology in patients with ischemic stroke [2,7-13]. However, in vivo imaging of neuroinflammation and gliosis is challenging [11, $12,14]$. One reason is that the astrocytes and microglia are highly dynamic and heterogeneous in their subtypes, locations, and activation status [15]. Additionally, the identification of an ideal target for neuroinflammation imaging is highly demanding. Translocator protein (TSPO) is the most widely used neuroinflammation target for PET imaging. $\left[{ }^{11} \mathrm{C}\right]$ PK-11195, the first-generation TSPO PET tracer, and several second-generation tracers such as $\left[{ }^{11} \mathrm{C}\right] \mathrm{DAA} 1106,\left[{ }^{11} \mathrm{C}\right]$ PBR06, $\left[{ }^{11} \mathrm{C}\right]$ PBR 28 , and $\left[{ }^{18} \mathrm{~F}\right]$ DPA-713, $\left[{ }^{18} \mathrm{~F}\right] \mathrm{DPA}-714$, and third-generation tracers $\left[{ }^{11} \mathrm{C}\right] \mathrm{GE} 180$, and $\left[{ }^{11} \mathrm{C}\right] \mathrm{ER} 176$ [16-27], have been evaluated in (pre-)clinical studies. PET imaging studies using TSPO tracers have shown increased brain uptake in post-stroke, although different time course and distribution were detected depending on the animal models and procedures. A faster time-course is observed in permanent compared to temporary ischemic stroke, and a higher increase of TSPO ligand uptake in the infarct core in temporary stroke than in peri-infarct areas in permanent stroke was reported $[12,15,20,28]$. Thus, the development of novel PET probes for visualizing alternative targets in neuroinflammation have received great attention in recent years [29-32].

Cannabinoid type 2 receptors $\left(\mathrm{CB}_{2} \mathrm{R}\right)$ are mainly expressed by immune cells including monocytes and macrophages. In the brain, $\mathrm{CB}_{2} \mathrm{Rs}$ are primarily found on microglia and have low expression levels under physiological conditions $[1,3$, 33]. Upregulation of brain $\mathrm{CB}_{2} \mathrm{R}$ expression is reported under acute inflammation such as ischemic stroke, and related to lesion extension in the penumbra and subsequent functional recovery [34]. Treatment with $\mathrm{CB}_{2} \mathrm{R}$ agonists has been shown to be neuroprotective and attenuates macrophage/ microglial activation in mouse models of cerebral ischemia [34-38]. $\mathrm{CB}_{2} \mathrm{R}$ was also upregulated in other brain diseases with involvement of inflammation/microglia under chronic inflammation in neurodegenerative diseases such as Alzheimer's disease [39-42] and senescence-accelerated models [43] associated with amyloid- $\beta$ deposits [33, 44-50].

Several structural scaffolds of $\mathrm{CB}_{2} \mathrm{R}$ PET tracers have recently been developed [51-55] including pyridine derivatives, oxoquinoline derivatives, thiazole derivatives [56, 57], oxadiazole derivatives [58], carbazole derivatives [59], imidazole derivative [60], and thiophene derivatives [61, 62]. In this study, our newly developed pyridine derivative $\left[{ }^{18}\right.$ F]RoSMA-18-d6 (Fig. 3b), which exhibited sub-nanomolar affinity and high selectivity towards $\mathrm{CB}_{2} \mathrm{R}(\mathrm{Ki}: 0.8 \mathrm{nM}$, $\mathrm{CB}_{2} \mathrm{R} / \mathrm{CB}_{1} \mathrm{R}>12^{\prime} 000$ ) [63], was selected for $\mathrm{CB}_{2} \mathrm{R}$-targeted neuroinflammation imaging.

The aim of the current study was to evaluate the novel $\mathrm{CB}_{2} \mathrm{R}$ tracer, $\left[{ }^{18} \mathrm{~F}\right] \mathrm{RoSMA}-18-\mathrm{d} 6$, in the transient middle cerebral artery occlusion (tMCAO) mouse models of focal cerebral ischemia [64-70] using microPET. In addition, $\left[{ }^{18} \mathrm{~F}\right]$ FDG was included for evaluation of the cerebral metabolic rate of glucose (CMRglc) and as a readily available radiotracer benchmark for neuroinflammation [71, 72]. Diffusion-weighted imaging (DWI) and $\mathrm{T}_{2}$-weighted imaging were performed for anatomical reference and for delineating the lesion in tMCAO mice.

\section{Methods}

\section{Radiosynthesis}

$\left[{ }^{18} \mathrm{~F}\right]$ RoSMA-18-d6 was synthesized by nucleophilic substitution of the tosylate precursor with $\left[{ }^{18} \mathrm{~F}\right] \mathrm{KF} /$ Kryptofix 222 in acetonitrile [63]. The crude product was purified by reverse phase semi-preparative high-performance liquid chromatography and formulated with 5\% ethanol in water for intravenous injection and for biological evaluations. In a typical experiment, a moderate radiochemical yield of $\sim 12 \%$ (decay corrected) was achieved with a radiochemical purity $>99 \%$. The molar activities ranged from 156 to $194 \mathrm{GBq} / \mu \mathrm{mol}$ at the end of synthesis. The identity of the final product was confirmed by comparison with the HPLC retention time of the non-radioactive reference compound by co-injection. $\left[{ }^{18} \mathrm{~F}\right]$ FDG was obtained from a routine clinical production from the University Hospital Zurich, Switzerland.

\section{Animals}

Twenty-four male C57BL/6 J mice were obtained from Janvier Labs (Le Genest-Saint-Isle, France). The mice were scanned at $8-10$ weeks of age (20-25 g body weight). Mice were randomly allocated to sham-operation $(n=10)$ or tMCAO $(n=14)$. Mice underwent MRI, $\mu$ PET/computed tomography (CT), and 2,3,5-triphenyltetrazolium chloride 
(TTC) histology staining for validation $24 \mathrm{~h}$ or $48 \mathrm{~h}$ after reperfusion. Animals were housed in ventilated cages inside a temperature-controlled room, under a 12-h dark/light cycle. Pelleted food (3437PXL15, CARGILL) and water were provided ad libitum. Paper tissue and red Tecniplast mouse house ${ }^{\circledR}$ (Tecniplast, Milan, Italy) shelters were placed in cages as environmental enrichments. All experiments were performed in accordance with the Swiss Federal Act on Animal Protection and were approved by the Cantonal Veterinary Office Zurich (permit number: ZH018/14 and ZH264/16).

Surgeries for tMCAO and sham-operation were performed using standard-operating procedures as described before $[73,74]$. Anesthesia was initiated by using $3 \%$ isoflurane (Abbott, Cham, Switzerland) in a 1:4 oxygen/air mixture and maintained at $2 \%$. Before the surgical procedure, a local analgesic (Lidocaine, $0.5 \%, 7 \mathrm{mg} / \mathrm{kg}$, Sintectica S.A., Switzerland) was administered subcutaneously (s.c.). Temperature was kept constant at $36.5 \pm 0.5^{\circ} \mathrm{C}$ with a feedback controlled warming pad system. All surgical procedures were performed in 15-30 min. After surgery, buprenorphine was administered as s.c. injection (Temgesic, $0.1 \mathrm{mg} / \mathrm{kg}$ b.w.), and at $4 \mathrm{~h}$ after reperfusion and supplied thereafter via drinking water $(1 \mathrm{~mL} / 32 \mathrm{~mL}$ of drinking water) until $24 \mathrm{~h}$ or $48 \mathrm{~h}$. Animals received softened chow in a weighing boat on the cage floor to encourage eating. tMCAO animals were excluded from the study if they met one of the following criteria: Bederson testing was performed $2 \mathrm{~h}$ post-reperfusion. Bederson score of 0 , no reflow after filament removal, and premature death.

\section{mRNA Isolation, Reverse-Transcription Reaction, and Real-Time Polymerase Chain Reaction}

Brain hemispheres of C57BL/6 mouse and tMCAO mice at $24 \mathrm{~h}$ and $48 \mathrm{~h}$ post reperfusion were used for total mRNA isolation according to the protocols of the Isol-RNA Lysis Reagent (5 PRIME, Gaithersburg, USA) and the bead-milling TissueLyser system (Qiagen, Hilden, Germany). QuantiTect ${ }^{\circledR}$ Reverse Transcription Kit (Qiagen, Hilden, Germany) was used to generate cDNA. The primers (Microsynth, Balgach, Switzerland) used for the quantitative polymerase chain reaction (qPCR) are summarized in Supplementary Table 1. Quantitation of CNR2, ionized calcium binding adaptor molecule 1 (Ibal), tumor necrosis factor (TNF-a), matrix metallopeptidase 9 (MMP9), glial fibrillary acidic protein (GFAP), and microtubule-associated protein 2 (MAP-2) mRNA expression was performed with the DyNAmo ${ }^{\mathrm{TM}}$ Flash SYBR ${ }^{\circ}$ Green qPCR Kit (Thermo Scientific, Runcorn, UK) using a 7900 HT Fast Real-Time PCR System (Applied Biosystems, Carlsbad, USA). The amplification signals were detected in real-time, which permitted accurate quantification of the amounts of the initial RNA template during 40 cycles according to the manufacturer's protocol. All reactions were performed in duplicates and in two independent runs. Quantitative analysis was performed using the SDS Software (v2.4) and a previously described $2^{-\Delta \Delta \mathrm{Ct}}$ quantification method [75]. The specificity of the PCR products of each run was determined and verified with the SDS dissociation curve analysis feature.

\section{In vivo $M R I$}

Data were acquired at $24 \mathrm{~h}$ after reperfusion on a $7 \mathrm{~T}$ Bruker Pharmascan (Bruker BioSpin GmbH, Germany), equipped with a volume resonator operating in quadrature mode for excitation and a four-element phased-array surface coil for signal reception and operated by Paravision 6.0 (Bruker BioSpin) $[73,76-78]$. Mice were anesthetized with an initial dose of $4 \%$ isoflurane in oxygen/air $(200: 800 \mathrm{ml} / \mathrm{min})$ and maintained at $1.5 \%$ isoflurane in oxygen/air $(100: 400 \mathrm{ml} /$ min). Body temperature was monitored with a rectal temperature probe (MLT415, ADInstruments) and kept at $36.5 \pm 0.5^{\circ} \mathrm{C}$ using a warm water circuit integrated into the animal support (Bruker BioSpin GmbH, Germany). $\mathrm{T}_{2}$-weighted $\mathrm{MR}$ images were obtained using a spin echo sequence (TurboRARE) with an echo time $3 \mathrm{~ms}$, repetition time $6 \mathrm{~ms}, 100$ averages, slice thickness $1 \mathrm{~mm}$, field-ofview $2.56 \mathrm{~cm} \times 1.28 \mathrm{~cm}$, matrix size $256 \times 128$, giving an in-plane resolution of $100 \mu \mathrm{m} \times 100 \mu \mathrm{m}$. For DWI, a fourshot spin echo-echo planar imaging sequence with an echo time $=28 \mathrm{~ms}$, repetition time $=3000[76,77]$, acquired with a field-of-view of $3.3 \mathrm{~cm} \times 2 \mathrm{~cm}$ and a matrix size of $128 \times 128$, resulting in a nominal voxel size of $258 \mu \mathrm{m} \times 156 \mu \mathrm{m}$. Diffusion-encoding was applied in the $\mathrm{x}-, \mathrm{y}-$, and $\mathrm{z}$-directions with $b$ values of $100,200,400,600,800$, and $1000 \mathrm{~s} / \mathrm{mm}^{2}$, respectively, acquisition time $3 \mathrm{~min} 48 \mathrm{~s}$. The ischemic lesion was determined as an area of significant reduction of the apparent diffusion coefficient (ADC) value compared with the unaffected contralateral side [79]. On $\mathrm{T}_{2}$-weighted images, the lesion was determined as an area of hyperintensities compared with the contralateral side.

\section{In vivo microPET Studies}

MicroPET/CT scans were performed at $24 \mathrm{~h}$ after reperfusion with a calibrated SuperArgus $\mu$ PET/CT scanner (Sedecal, Madrid, Spain) with an axial field-of-view of $4.8 \mathrm{~cm}$ and a spatial resolution of 1.6-1.7 $\mathrm{mm}$ (full width at half maximum). tMCAO and the sham-operated C57BL/6 J mice were anesthetized with ca. $2.5 \%$ isoflurane in oxygen/air (1:1) during tracer injection and the whole scan time period. The formulated radioligand solution $\left(\left[{ }^{18} \mathrm{~F}\right] \mathrm{FDG}\right.$ : $9.9-11 \mathrm{MBq}$ or $\left[{ }^{18} \mathrm{~F}\right]$ RoSMA-18-d6: 7.2-13 MBq) was administered via tail vein injection, and mice were dynamically scanned for $60 \mathrm{~min}$. For blocking experiments, $1.5 \mathrm{mg} / \mathrm{kg} \mathrm{GW} 405833$ (Fig. 3a) was dissolved in a vehicle of $2 \%$ Cremophor (v/v), $10 \%$ ethanol (v/v), and $88 \%$ water for injection (v/v) and injected together with $\left[{ }^{18} \mathrm{~F}\right]$ RoSMA-18-d6. Body temperature was monitored by a rectal probe and kept at $37^{\circ} \mathrm{C}$ by a heated air stream $\left(37^{\circ} \mathrm{C}\right)$. The anesthesia depth was measured 

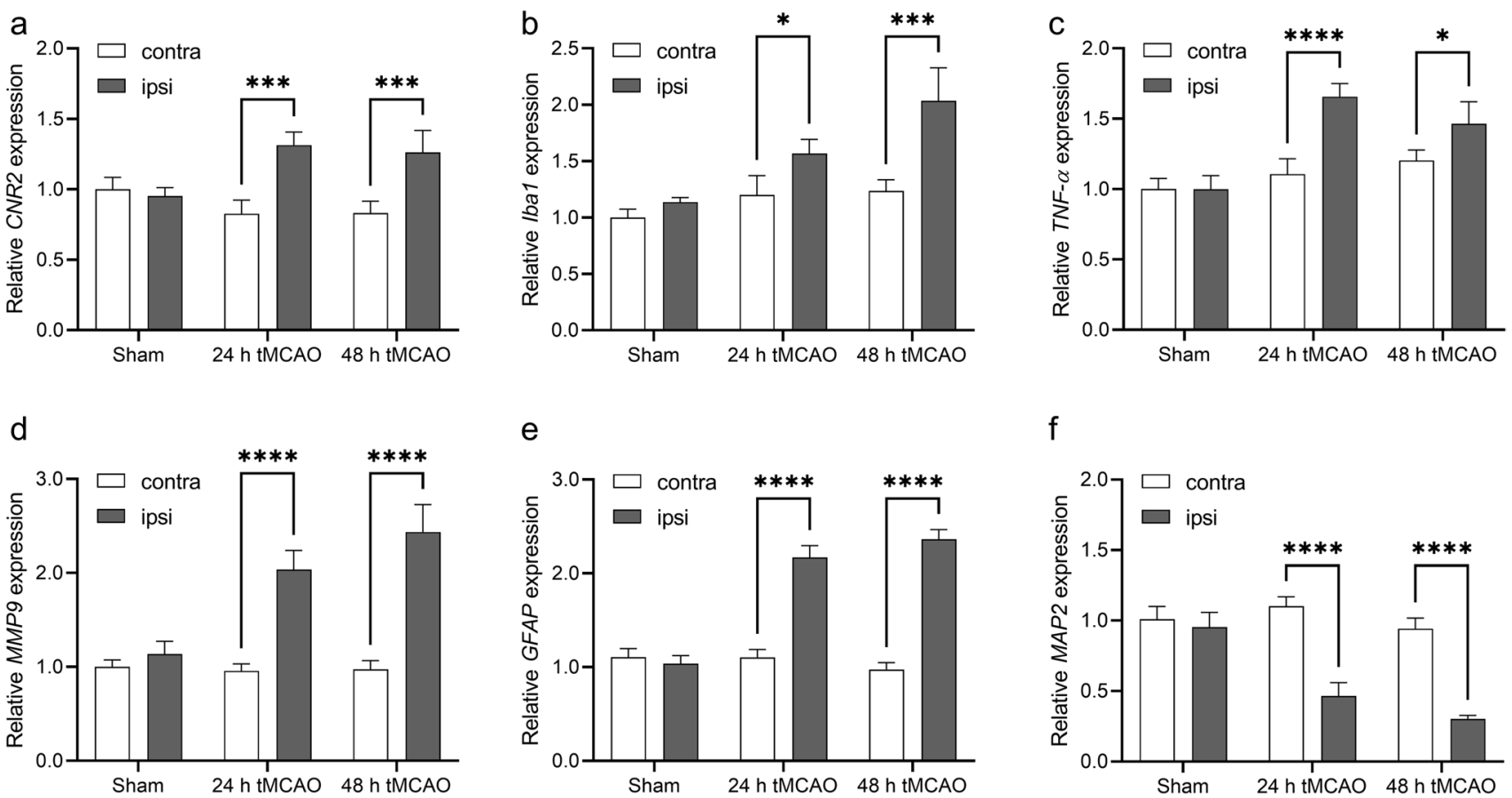

Fig. 1. Relative mRNA levels of inflammatory markers and neuronal damage in sham-operated and tMCAO mouse brain in contra-and ipsilateral brain hemisphere at $24 \mathrm{~h}$ and $48 \mathrm{~h}$ after reperfusion. a CNR2, b Iba1, c TNF- $a, \mathbf{d} M M P 9$, e GFAP, and f MAP-2. Values represent mean \pm standard deviation. Expression levels were quantified by qPCR relative to $\beta$-actin. ${ }^{*} p<0.05,{ }^{* * *} p<0.001,{ }^{* * * *} p<0.0001$.

by the respiratory frequency (SA Instruments, Inc., Stony Brook, USA). $\mu$ PET acquisitions were combined with CT for anatomical orientation and attenuation correction. The obtained data were reconstructed in user-defined time frames with a voxel size of $0.3875 \times 0.3875 \times 0.775 \mathrm{~mm}^{3}$ as previously described [80].

\section{Triphenyltetrazolium Chloride (TTC) Staining}

To assess the ischemic lesion severity in the brain of tMCAO mice and to validate the absence of lesion in the shamoperated mice, staining with TTC staining was performed. After measurements mice were euthanized, their brains were removed and 1-mm thick brain slices were obtained with a brain matrix. Slices were incubated in a $2.5 \%$ TTC solution (Sigma-Aldrich, Switzerland) in PBS at $37{ }^{\circ} \mathrm{C}$ for 3 min. Photographs of the brain sections were taken. Edema-corrected lesion volumes were quantified as described [81].

\section{Biodistribution Studies in the Mouse Brain}

After PET/CT scanning of tMCAO mice at $24 \mathrm{~h}$ after reperfusion with $\left[{ }^{18} \mathrm{~F}\right]$ RoSMA-18-d6, animals were sacrificed at 70 min post injection by decapitation. The spleen and brain regions of ischemic ipsilateral area and contralateral hemisphere were collected for analysis with a gamma counter. The accumulated radioactivities in the different tissues were expressed as percent normalized injected dose per gram of tissue normalized to $20 \mathrm{~g}$ body weight of the animals (norm. percentage injected dose per gram tissue (\% ID/g tissue)).

\section{Data Analysis and Statistics}

Images were processed and analyzed using PMOD 4.2 software (PMOD Technologies Ltd., Zurich, Switzerland). The time - activity curves were deduced from specific volume-ofinterest that were defined based on a mouse MRI $\mathrm{T}_{2}$-weighted image template [82]. Radioactivity is presented as standardized uptake value (SUV) (decay-corrected radioactivity per $\mathrm{cm}^{3}$ divided by the injected dose per gram body weight). $\left[{ }^{18} \mathrm{~F}\right]$ RoSMA-18-d6 SUVR was calculated by using the midbrain in the corresponding hemisphere as reference brain region. For $\left[{ }^{18}\right.$ F]FDG PET, regional SUV was calculated. Two-way ANOVA with Sidak post hoc analysis was used for comparison between groups (Graphpad Prism 9.0, CA, USA).

\section{Results}

Increased expression of inflammation makers and neuronal damage after focal cerebral ischemia in tMCAO mice mRNA levels were measured to address the question whether mouse non-ischemic and ischemic hemispheres differ in their expression levels of $C N R 2$ and other inflammatory genes. CNR2 mRNA expression was increased to around 1.3-fold after $24 \mathrm{~h}$ reperfusion and at $48 \mathrm{~h}$ in the ipsilateral comparing to contralateral hemisphere (Fig. 1a). Similarly, 1.5-2.5fold increases were observed in the mRNA expression of inflammatory markers including $T N F-\alpha, I b a l, M M P 9$, and $G F A P$ at $24 \mathrm{~h}$ and $48 \mathrm{~h}$ after reperfusion in the ipsilateral compared to contralateral brain region (Fig. 1b-e). MAP-2 
expression has been shown to be a reliable marker of neurons that undergo cell death [83, 84]. The neuron-specific $M A P-2$ expression was markedly reduced in the ipsilateral compared to contralateral hemisphere at $24 \mathrm{~h}$ and $48 \mathrm{~h}$ after reperfusion (Fig. 1f). As similar CNR2 mRNA expression was observed at $24 \mathrm{~h}$ and $48 \mathrm{~h}$, our studies were performed at early time point of $24 \mathrm{~h}$ after reperfusion for investigating the functional, structural, and molecular changes in the following experiments.

\section{Reduced Cerebral Glucose Metabolism and Structural MRI Lesion Following tMCAO}

Reduced $\left[{ }^{18} \mathrm{~F}\right] \mathrm{FDG}$ uptake was observed in the presumed MCA territory of the ipsilateral hemisphere in tMCAO mice, while there was no difference in $\left[{ }^{18} \mathrm{~F}\right] \mathrm{FDG}$ uptake between hemispheres in sham-operated mice (Fig. 2a). SUVs were significantly lower in the ipsilateral striatum in tMCAO compared to the contralateral side and compared to the same region in sham-operated mice 1.8 vs 1.4 (Fig. 2b). There were no differences in $\left[{ }^{18} \mathrm{~F}\right] \mathrm{FDG}$ uptake in the cortex and cerebellum between the ipsilateral and contralateral hemisphere in tMCAO mice and sham-operated mice. $\mathrm{T}_{2}$-weighted MRI and
DWI imaging were performed in $\mathrm{TMCAO}$ and sham-operated animals at $24 \mathrm{~h}$ after reperfusion (Fig. 2c). The lesions in the ipsilateral side in the striatum and cortex were visible as areas of decreased values on the ADC maps calculated from DWI and as areas of increased intensities on the $\mathrm{T}_{2}$-weighted MR images at $24 \mathrm{~h}$ after reperfusion following $1 \mathrm{~h}$ tMCAO (Figs. 2c-d). Ischemic lesions in the tMCAO were also seen ex vivo as white areas, while viable tissue appeared red in TTC stained brain sections (Fig. 2e). Homogenous deep red color was observed across both hemispheres in sham-operated mice, verifying the absence of any lesion. The hemispheric lesion volumes in tMCAO mice were $42.8 \pm 10.2 \%$ (mean \pm standard deviation).

\section{Increased $\left[{ }^{18}\right.$ F $]$ RoSMA-18-d6 Retention in the Striatum After $\mathrm{IMCAO}$}

To analyze the distribution of $\left[{ }^{18} \mathrm{~F}\right] \mathrm{RoSMA}-18$-d6 in tMCAO mice brain, dynamic $\mu$ PET/CT scans were performed at $24 \mathrm{~h}$ after reperfusion. The standard uptake values (SUVs) of $\left[{ }^{18} \mathrm{~F}\right]$ RoSMA-18-d6 did not reveal significant difference in various brain regions of tMCAO mice (Supplementary Fig. 1). However, we found a reduced uptake at early time frame a

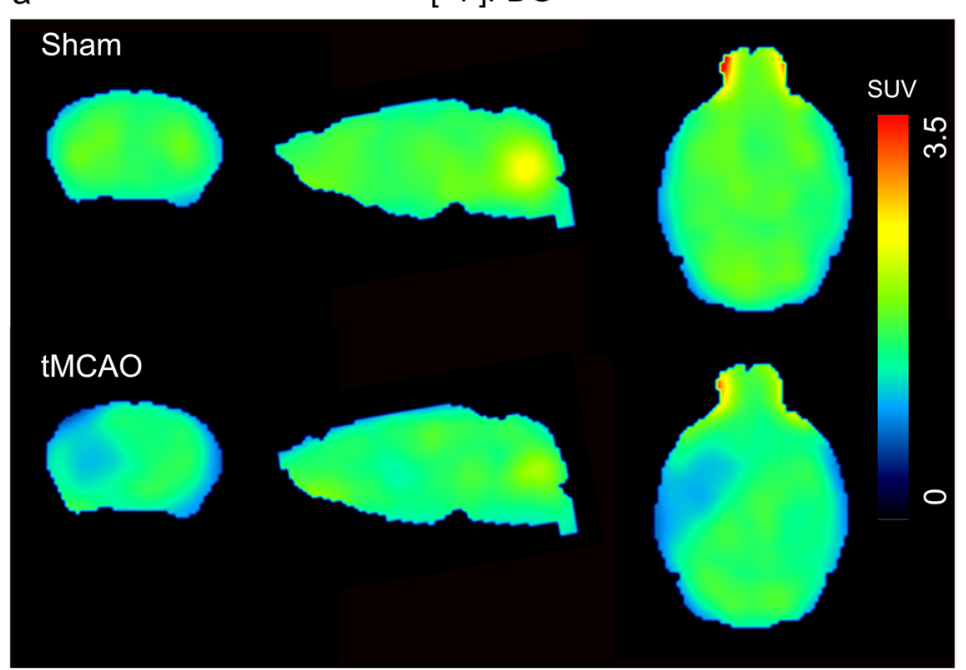

C T2 $\quad d \quad A D C$

e

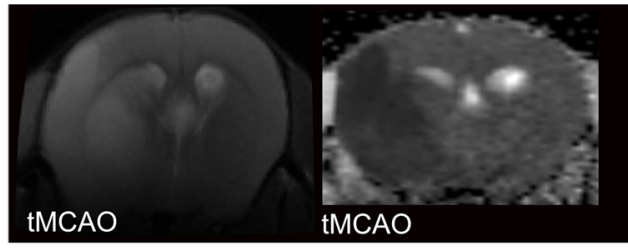

b

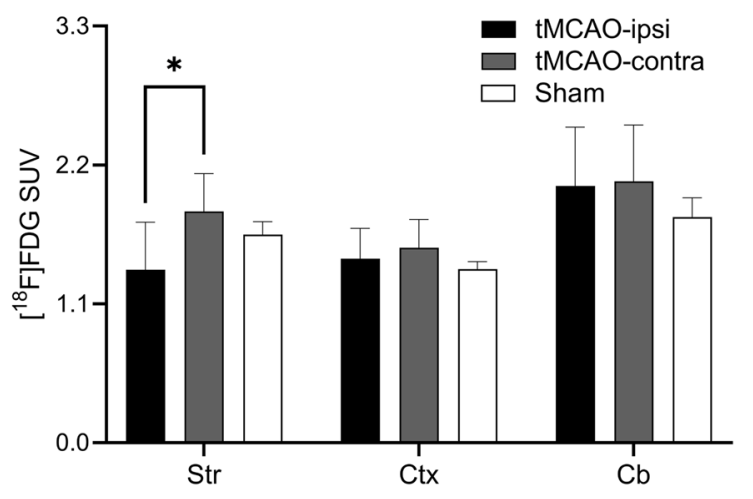

TTC
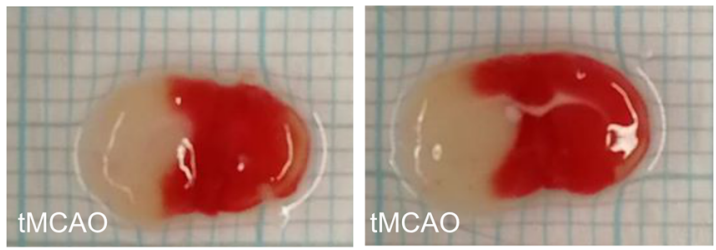

Fig. 2. In vivo $\mathrm{MRI}$ and $\left[{ }^{18} \mathrm{~F}\right] \mathrm{FDG}$ PET in $\mathrm{TMCAO}$ mouse brain. a Representative PET images of coronal, sagittal and horizontal mouse brain sections after intravenous injection of $\left[{ }^{18} \mathrm{~F}\right] \mathrm{FDG}$ in sham-operated and $\mathrm{tMCAO}$ mice. The radiosignals were averaged from 21 to $61 \mathrm{~min} ; \mathbf{b}\left[{ }^{18} \mathrm{~F}\right]$ FDG accumulation (SUV) at different mouse brain regions (Str: striatum; Ctx: cortex; Cb: cerebellum) in sham and tMCAO mice. Significantly reduced $\left[{ }^{18} \mathrm{~F}\right] \mathrm{FDG}$ accumulation was observed in the ipsilateral striatum compared to contralateral side in tMCAO mice; c-e in vivo $\mathrm{T}_{2}$-weighted image, ADC map, and ex vivo TTC stained brain sections, indicating the delineation in tMCAO mice. TTC, 2,3,5-triphenyltetrazolium chloride; ADC, apparent diffusion coefficient; SUV, standard uptake value. ${ }^{*} p<0.05$. 
Fig. 3. In vivo microPET imaging of tMCAO mouse brain using $\left[{ }^{18} \mathrm{~F}\right]$ RoSMA-18-d6. a,b Chemical structures of blocker GW405833 and $\left[{ }^{18} \mathrm{~F}\right]$ RoSMA-18-d6; c representative PET images of horizontal mouse brain sections at different time frames after intravenous injection of $\left[{ }^{18} \mathrm{~F}\right] \mathrm{RoSMA}-$ 18-d6; SUV: 0-0.5; d,e ratios of $\left[{ }^{18}\right.$ F]RoSMA-18-d6 uptake under baseline and blockade conditions in cortex and striatum. Significantly higher $\left[{ }^{18} \mathrm{~F}\right]$ RoSMA-18-d6 standard uptake value ratio (SUVR) was observed in the ischemic ipsilateral striatum under baseline conditions, but not in the ipsilateral cortex. Midbrain was used as reference brain region for SUVR calculation. ${ }^{*} p<0.05$. a

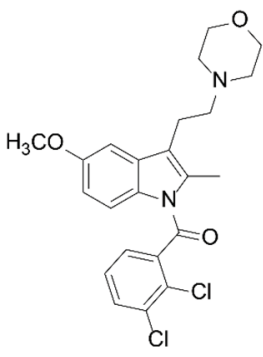

GW 405833

(CB2 selective agonist) b

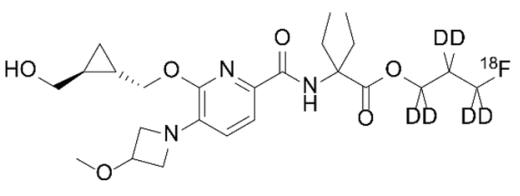

$\left[{ }^{18}\right.$ F]RoSMA-18-d6

C

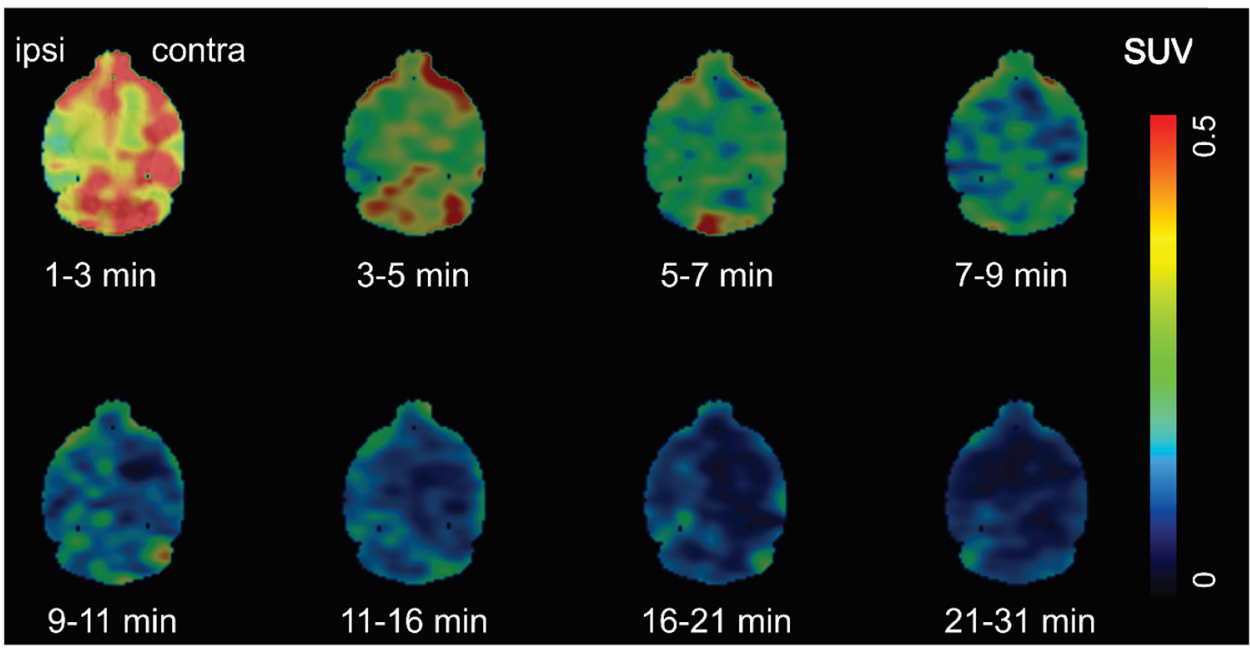

d

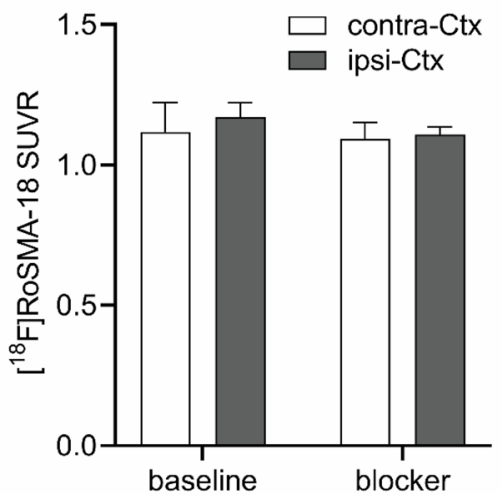

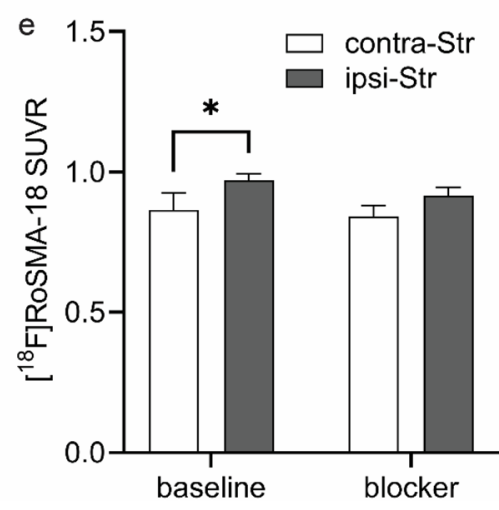

(1-3 $\mathrm{min})$ and a similar uptake after $7 \mathrm{~min}$ in the ipsilateral side compared to that of the contralateral side (Fig. 3c, and whole head images in Supplementary Fig. 2). Thus, to exclude the perfusion influence, we averaged the brain signals from 21-61 min and selected the midbrain as the reference region. Higher $\left[{ }^{18} \mathrm{~F}\right]$ RoSMA-18-d6 SUVR was observed in the ischemic ipsilateral striatum compared to the contralateral striatum (two-way ANOVA with Sidak multiple comparison correction, $0.97 \pm 0.02$ vs $0.87 \pm 0.06, p=0.0274)$, but not in other brain regions such as cortex (Figs. 3d, e). The increased signals at ischemic ipsilateral striatum, however, could not be blocked by the selective $\mathrm{CB}_{2} \mathrm{R}$ agonist GW405833 (Fig. 3e).

At the end of the in vivo experiments, we dissected the mice to verify the activity accumulation and specificity of $\left[{ }^{18} \mathrm{~F}\right]$ RoSMA-18-d6 in the spleen and different brain regions with a gamma counter. In line with the results obtained from the averaged SUVRs in the tMCAO mouse brain, the radioactivity in the ipsilateral side was indeed significantly higher than that of the contralateral hemisphere $(0.037 \pm 0.007$ vs $0.026 \pm 0.003, n=5$ each group $)$, but no blockade effect was seen under blocking conditions (Fig. 4a). As expected, radioactivity in the $\mathrm{CB}_{2} \mathrm{R}$-rich spleen was much higher than the brain and $58 \%$ of the signals was blocked by co-injection of $\mathrm{CB}_{2} \mathrm{R}$ specific ligand GW405833, demonstrating specific target engagement of $\left[{ }^{18} \mathrm{~F}\right]$ RoSMA-18-d6 in vivo (Fig. $4 \mathrm{~b}$ ). 

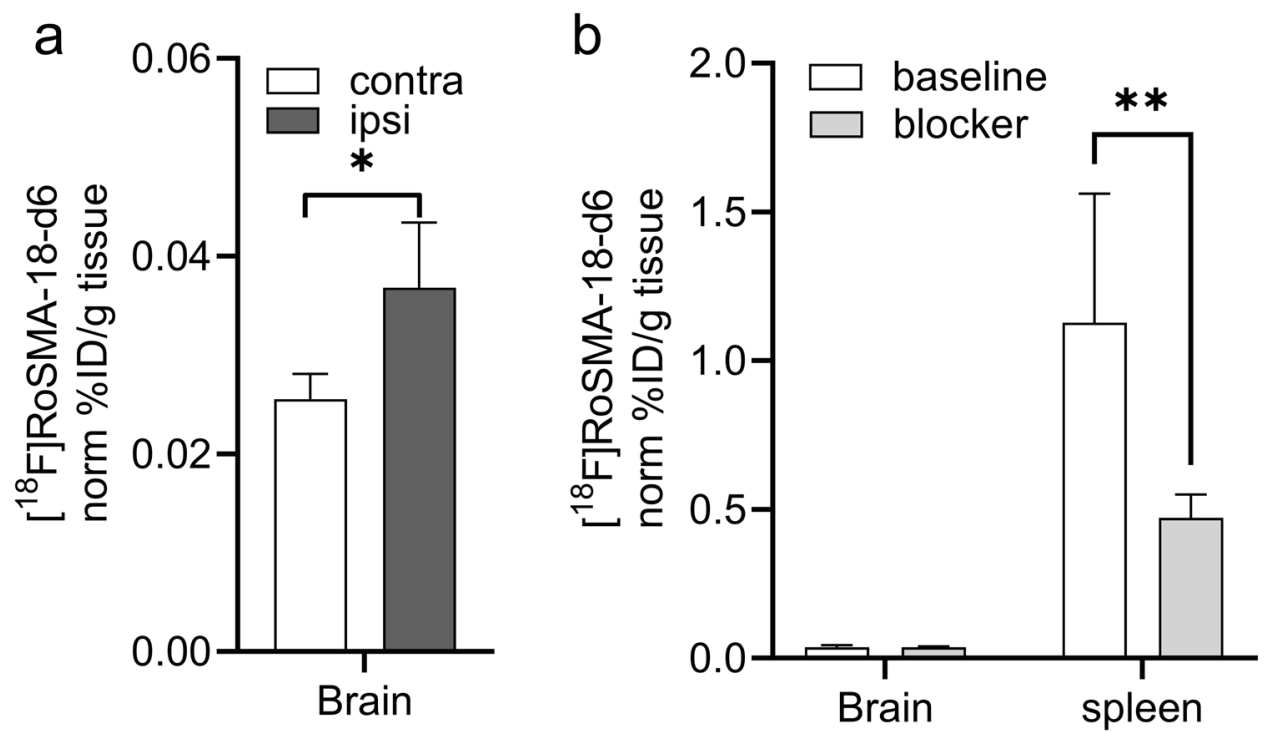

Fig. 4. Ex vivo biodistribution of $\left[{ }^{18} \mathrm{~F}\right]$ RoSMA-18-d6 in the brain and spleen of tMCAO mouse. Animals $(n=4)$ were sacrificed at 70 min postinjection, and the spleen and brain regions were dissected and analyzed with a gamma counter. a Higher $\left[{ }^{18} \mathrm{~F}\right]$ RoSMA-18-d6 binding (norm $\%$ ID/g tissue) was detected in the ipsilateral vs contralateral hemisphere under baseline conditions. $\mathbf{b}$ In the spleen about $58 \%$ of the $\left[{ }^{18} \mathrm{~F}\right]$ RoSMA-18-d6 binding (norm \% ID/g tissue) was blocked. No significant blocking was observed in the brain. Data are presented as the mean of the percentage of injected dose per gram tissue normalized to $20 \mathrm{~g}$ body weight; mean \pm standard deviation. \% ID/g: percentage injected dose per gram. ${ }^{*} p<0.05,{ }^{* *} p<0.01$.

\section{Discussion}

This study assessed the utility of $\mathrm{CB}_{2} \mathrm{R}$ PET tracer $\left[{ }^{18} \mathrm{~F}\right]$ RoSMA-18-d6 for imaging tMCAO mouse at subacute stage, concomitant with decreased CMRglc levels and formation of a structural lesion. Previous PET imaging of stroke animal models led to inconclusive results. In a rat model of photothrombotic stroke at $24 \mathrm{~h}$ after surgery, increased $\left[{ }^{11} \mathrm{C}\right] \mathrm{NE} 40$ $\left(\mathrm{CB}_{2} \mathrm{R}\right.$ tracer) uptake and unvaried $\left[{ }^{11} \mathrm{C}\right] \mathrm{PK} 11195$ (TSPO tracer) uptake were reported [85]. In another study, $\left[{ }^{11} \mathrm{C}\right]$ NE40 uptake did not show any difference in the same rat model of photothrombotic stroke [86]. Moreover, reduced $\left[{ }^{11} \mathrm{C}\right] \mathrm{A} 836339\left(\mathrm{CB}_{2} \mathrm{R}\right.$ tracer$)$ uptake was reported in a focal tMCAO rat model over 1-28 days after occlusion [56]. Possible reasons for these different observations include the time point of assessment, different methods for inducing acute stroke (transient or permanent ischemia) resulting in variations of ischemic severity and levels of inflammatory cell expression [36].

$\mathrm{CB}_{2} \mathrm{R}$ has negligible expression in the mouse brain and is mainly expressed in the spleen under physiological conditions [39, 45, 64-69, 87]. Under neuroinflammatory conditions, $\mathrm{CB}_{2} \mathrm{R}$ is upregulated in activated microglial cells. In this study, we used quantitative real-time polymerase chain reaction to measure gene expression levels of $C N R 2, T N F$ $\alpha, I b a 1, M M P 9, G F A P$, and MAP-2 at $24 \mathrm{~h}$ and $48 \mathrm{~h}$. All tested inflammatory markers displayed increased mRNA levels in the ipsilateral brain hemisphere, in agreement with the reported findings in tMCAO mouse model [34, 38, 88, 89]. In line with the increased CNR2 gene expression levels, significantly higher $\left[{ }^{18} \mathrm{~F}\right]$ RoSMA-18-d6 SUVR (standard uptake value ratio) was observed in striatum at ipsilateral vs contralateral under baseline conditions in our PET studies. The $50 \%$ reduction of the neuronal marker $M A P-2$ indicated neuronal damage.

The dynamic $\mu$ PET scan using $\left[{ }^{18} \mathrm{~F}\right]$ RoSMA-18-d6 indicated a reduced perfusion in the lesion brain regions at the first time frame of $1-3 \mathrm{~min}$. This is probably due to the changes of microvascular response (no-reflow phenomenon) and the reduction in neuronal activity. Taking the midbrain as the reference region, the ratios of SUV averaged from 21 to $61 \mathrm{~min}$ revealed increased $\left[{ }^{18} \mathrm{~F}\right] \mathrm{RoSMA}-$ 18-d6 SUVR in the ipsilateral ischemic striatum compared to that of the contralateral side. Our ex vivo bio-distribution studies confirmed the difference of the radioactivity distribution in the left and right brain hemisphere. The in vivo specificity of $\left[{ }^{18} \mathrm{~F}\right] \mathrm{RoSMA}-18$-d6 towards $\mathrm{CB}_{2} \mathrm{R}$ is evidenced by a $58 \%$ reduction in radioactivity in the mouse spleen under blockade conditions in ex vivo biodistribution studies. Underlying reasons for the lack of specificity of $\left[{ }^{18} \mathrm{~F}\right]$ RoSMA-18-d6 in the mouse brain may include (1) the increased tracer availability in the blood induced by blocking the $\mathrm{CB}_{2} \mathrm{R}$ peripheral targets in the presence of the blocker GW405833; and (2) the relatively low brain uptake of our $\mathrm{CB}_{2} \mathrm{R}$-selective radioligand $\left[{ }^{18} \mathrm{~F}\right]$ RoSMA-18-d6 in the mouse brain resulted in undetectable changes of radiosignals under baseline and blockade conditions. Notably, the time-activity curves of $\left[{ }^{18} \mathrm{~F}\right]$ RoSMA18-d6 in tMCAO mouse brain showed remarkably higher initial brain uptake under blockade conditions than the baseline in both sides of the mouse brain (Supplementary Fig. 1), indicating the influence of blocking $\mathrm{CB}_{2} \mathrm{R}$ target 
in the peripheral organs on the availability of radiotracer concentrations in the blood. In our previous studies with Wistar rats, the spleen uptake of $\left[{ }^{18} \mathrm{~F}\right]$ RoSMA-18-d6 was blocked by nearly $90 \%$. The higher specific binding of $\left[{ }^{18} \mathrm{~F}\right]$ RoSMA-18-d6 in rat spleen is likely due to the increased available targets. Govaerts et al. reported higher binding sites $\mathrm{B}_{\max }$ in the rat spleen $(0.71 \pm 0.02 \mathrm{pmol} / \mathrm{mg}$ protein $)$ compared to that in the mouse spleen $(0.31 \pm 0.03 \mathrm{pmol} / \mathrm{mg}$ protein) by using $\left[{ }^{3} \mathrm{H}\right] \mathrm{CP} 55940$ binding assay [90]. In the mouse P-glycoprotein assay, compound RoSMA-18 exhibits an efflux ratio (ER) of 4.6, which partly explains the low initial brain uptake in mice. Compared to other $\mathrm{CB}_{2} \mathrm{R}$ radioligand developed in our group, $\left[{ }^{18} \mathrm{~F}\right] \mathrm{ROSMA}-18-\mathrm{d} 6$ has the highest specificity $(86 \%)$ towards $\mathrm{CB}_{2} \mathrm{R}$ based on the ex vivo biodistribution results in the rat spleen and was therefore selected for in vivo studies.

We observed that $\left[{ }^{18} \mathrm{~F}\right] \mathrm{FDG}$ measure of CMRglc was reduced in the ischemic areas, i.e., ipsilateral striatum of the tMCAO mice at $24 \mathrm{~h}$ after reperfusion. The reduced CMRglc was reported in many earlier studies in disease animal models and in stroke patients, [91-94], masking CMRglc reduction of neuronal tissue in the brain. At an extended time points of the recovery stage from days 4 to 40 , an increased CMRglc level was reported in the ischemic regions due to the increased consumption from inflammatory cells along with microglial activation [91, 95, 96].

There are several limitations in the current study. (1) As there is no reliable specific $\mathrm{CB}_{2} \mathrm{R}$ antibody, we did not include immunohistochemical staining for $\mathrm{CB}_{2} \mathrm{R}$ protein distribution in the mouse brain. The qPCR measures of CNR2 mRNA level provided an alternative readout, but do not provide spatial distribution of cerebral $\mathrm{CB}_{2} \mathrm{R}$ expression. (2) Due to the logistic barrier, MRI and $\mu \mathrm{PET} /$ CT scans were performed with different cohorts of animals. Nevertheless, standard operating procedures for the surgery were used. (3) Our in vivo data with tMCAO mice were collected at $24 \mathrm{~h}$ after surgery, longitudinal imaging of tMCAO mice with $\left[{ }^{18} \mathrm{~F}\right]$ RoSMA-18-d6 along with structural and functional readout will provide further insight into the spatio-temporal dynamics of $\mathrm{CB}_{2} \mathrm{R}$ expression in the brain. Moreover, $\left[{ }^{18} \mathrm{~F}\right] \mathrm{FDG}-\mathrm{PET}$ was used as a benchmark for neuroinflammation in the current study. Further study using TSPO tracer to assess the glial activation along with $\mathrm{CB}_{2} \mathrm{R}$ tracer will provide valuable insights.

\section{Conclusion}

Our newly developed $\mathrm{CB}_{2} \mathrm{R}$ PET tracer, $\left[{ }^{18} \mathrm{~F}\right]$ RoSMA-18-d6, revealed limited utility to image neuroinflammation in the ischemic ipsilateral of the tMCAO mice at $24 \mathrm{~h}$ after reperfusion. Although lesion regions in tMCAO mouse brain could be followed by the ratios of averaged SUVs from 21 to $61 \mathrm{~min}$ with midbrain as the reference region, the in vivo specificity of $\left[{ }^{18} \mathrm{~F}\right]$ RoSMA-18-d6 was confirmed only in the $\mathrm{CB}_{2} \mathrm{R}$-rich spleen. Different neuroinflammatory animal models which have comparable neuronal numbers in the lesion regions are recommended for evaluation of $\mathrm{CB}_{2} \mathrm{R}$ in further PET imaging studies.

Supplementary Information The online version contains supplementary material available at https://doi.org/10.1007/s11307-021-01655-4.

Acknowledgements The authors acknowledge Yingfang He and Annette Krämer at Center for Radiopharmaceutical Sciences, Department of Chemistry and Applied Biosciences, ETH Zurich, and Dr Mark Augath, at the Institute for Biomedical Engineering, ETH Zurich, and University of Zurich, for technical assistance.

Author Contributions RN, JK, LM, and SMA designed the study; RN, KC, $\mathrm{AH}, \mathrm{AMH}, \mathrm{GL}$, and LM performed the experiment; RN and LM performed data analysis; RN, JK, and LM wrote the initial manuscript. All authors read and approved the final manuscript.

Funding Open access funding provided by Swiss Federal Institute of Technology Zurich. JK received funding from the Swiss National Science Foundation (320030_179277), in the framework of ERA-NET NEURON (32NE30_173678/1), the Synapsis Foundation, the Olga Mayenfisch Stiftung, and the Vontobel foundation. RN received funding from Forschungskredit, Synapsis foundation career development award (2017 CDA-03), and the Vontobel foundation.

\section{Declarations}

Conflict of interest The authors declare that they have no conflicts of interest.

Open Access This article is licensed under a Creative Commons Attribution 4.0 International License, which permits use, sharing, adaptation, distribution and reproduction in any medium or format, as long as you give appropriate credit to the original author(s) and the source, provide a link to the Creative Commons licence, and indicate if changes were made. The images or other third party material in this article are included in the article's Creative Commons licence, unless indicated otherwise in a credit line to the material. If material is not included in the article's Creative Commons licence and your intended use is not permitted by statutory regulation or exceeds the permitted use, you will need to obtain permission directly from the copyright holder. To view a copy of this licence, visit http://creativeco mmons.org/licenses/by/4.0/.

\section{References}

1. Astrup J (1982) Energy-requiring cell functions in the ischemic brain. Their critical supply and possible inhibition in protective therapy. J Neurosurg 56:482-497

2. Heiss W-D (2000) Ischemic penumbra: evidence from functional imaging in man. J Cereb Blood Flow Metab 20:1276-1293

3. Dirnagl U, Iadecola C, Moskowitz MA (1999) Pathobiology of ischaemic stroke: an integrated view. Trends Neurosci 22:391-397

4. Dabrowska S, Andrzejewska A, Lukomska B, Janowski M (2019) Neuroinflammation as a target for treatment of stroke using mesenchymal stem cells and extracellular vesicles. J Neuroinflamm 16:178

5. Anrather J, Iadecola C (2016) Inflammation and stroke: an overview. Neurotherapeutics 13:661-670

6. Lambertsen KL, Finsen B, Clausen BH (2019) Post-stroke inflammation-target or tool for therapy? Acta Neuropathol 137:693-714

7. Evans NR et al (2017) PET imaging of the neurovascular interface in cerebrovascular disease. Nat Rev Neurol 13:676-688 
8. Marchal G et al (1993) PET imaging of cerebral perfusion and oxygen consumption in acute ischaemic stroke: relation to outcome. Lancet 341:925-927

9. Muir KW, Buchan A, von Kummer R, Rother J, Baron J-C (2006) Imaging of acute stroke. Lancet Neurol 5:755-768

10. Carter SF et al (2019) Astrocyte biomarkers in Alzheimer's disease Trends Mol Med 25:77-95

11. Jain P et al (2020) Neuroinflammation PET imaging: current opinion and future directions. J Nucl Med 61:1107-1112

12. Zinnhardt B et al (2018) In vivo imaging biomarkers of neuroinflammation in the development and assessment of stroke therapies - towards clinical translation. Theranostics 8:2603-2620

13. Martín A et al (2012) Imaging of perfusion, angiogenesis, and tissue elasticity after stroke. J Cereb Blood Flow Metab 32:1496-1507

14. Venneti S, Lopresti BJ, Wiley CA (2013) Molecular imaging of microglia/macrophages in the brain. Glia 61:10-23

15. Kreisl WC et al (2020) PET imaging of neuroinflammation in neurological disorders. The Lancet Neurology 19:940-950

16. Thiel A, Heiss WD (2011) Imaging of microglia activation in stroke. Stroke 42:507-512

17. Boutin $\mathrm{H}$ et al (2015) 18F-GE-180: a novel TSPO radiotracer compared to 11C-R-PK11195 in a preclinical model of stroke. Eur J Nucl Med Mol Imag 42:503-511

18. Lartey FM et al (2014) PET Imaging of stroke-induced neuroinflammation in mice using [18F]PBR06. Mol Imag Biol 16:109-117

19. Tóth $\mathrm{M}$ et al (2016) Acute neuroinflammation in a clinically relevant focal cortical ischemic stroke model in rat: longitudinal positron emission tomography and immunofluorescent tracking. Brain Struct Funct 221:1279-1290

20. Chaney A, et al (2018) 11C-DPA-713 versus 18F-GE-180: a preclinical comparison of TSPO-PET tracers to visualize acute and chronic neuroinflammation in a mouse model of ischemic stroke. J Nucl Med

21. Martín A et al (2009) Evaluation of the PBR/TSPO radioligand [18F] DPA-714 in a rat model of focal cerebral ischemia. J Cereb Blood Flow Metab 30:230-241

22. Thiel A et al (2010) The temporal dynamics of poststroke neuroinflammation: a longitudinal diffusion tensor imaging-guided pet study with ${ }^{11}$ C-PK11195 in acute subcortical stroke. J Nucl Med 51:1404-1412

23. Zinnhardt B et al (2015) Multimodal imaging reveals temporal and spatial microglia and matrix metalloproteinase activity after experimental stroke. J Cereb Blood Flow Metab 35:1711-1721

24. Ishikawa A et al (2018) In vivo visualization of Tau accumulation, microglial activation, and brain atrophy in a mouse model of tauopathy rTg4510. J Alzheimer's Dis 61:1037-1052

25. Zhou R et al (2021) PET imaging of neuroinflammation in Alzheimer's disease. Front Immunol 12:739130

26. Zanotti-Fregonara $P$ et al (2019) Head-to-head comparison of (11) C-PBR28 and (11)C-ER176 for quantification of the translocator protein in the human brain. Eur J Nucl Med Mol Imaging 46:1822-1829

27. Ni R et al (2021) In vitro characterization of the regional binding distribution of amyloid PET tracer florbetaben and the glia tracers deprenyl and PK11195 in autopsy Alzheimer's brain tissue. J Alzheimers Dis 80:1723-1737

28. Boutin H, Pinborg LH (2015) TSPO imaging in stroke: from animal models to human subjects. Clin Transl Imaging 3:423-435

29. Razansky D, Klohs J, Ni R (2021) Multi-scale optoacoustic molecular imaging of brain diseases. Eur J Nucl Med Mol Imaging

30. Ling $X$ et al (2015) A novel near-infrared fluorescence imaging probe that preferentially binds to cannabinoid receptors CB2R over CB1R. Biomaterials 57:169-178

31. Sarott RC et al (2020) Development of high-specificity fluorescent probes to enable cannabinoid type 2 receptor studies in living cells. J Am Chem Soc 142:16953-16964

32. Shi X-F et al (2021) Multimodal contrast agents for optoacoustic brain imaging in small animals. Front Bioeng Biotechnol 9:746815

33. Stempel AV et al (2016) Cannabinoid type 2 receptors mediate a cell type-specific plasticity in the hippocampus. Neuron 90:795-809

34. Zarruk JG et al (2012) Cannabinoid type 2 receptor activation downregulates stroke-induced classic and alternative brain macrophage/microglial activation concomitant to neuroprotection. Stroke 43:211-219

35. Ronca RD et al (2015) A selective cannabinoid CB2 agonist attenuates damage and improves memory retention following stroke in mice. Life Sci 138:72-77
36. Bravo-Ferrer I et al (2017) Cannabinoid type-2 receptor drives neurogenesis and improves functional outcome after stroke. Stroke 48:204-212

37. Zhang $\mathrm{M}$ et al (2007) Cannabinoid $\mathrm{CB}(2)$ receptor activation decreases cerebral infarction in a mouse focal ischemia/reperfusion model. J Cereb Blood Flow Metab 27:1387-1396

38. Yu SJ, et al (2015) Time-dependent protection of CB2 receptor agonist in stroke. PLOS ONE 10:e0132487

39. López A et al (2018) Cannabinoid CB2 receptors in the mouse brain: relevance for Alzheimer's disease. J Neuroinflamm 15:158

40. Rodriguez-Vieitez E et al (2015) Astrocytosis precedes amyloid plaque deposition in Alzheimer APPswe transgenic mouse brain: a correlative positron emission tomography and in vitro imaging study. Eur J Nucl Med Mol Imaging 42:1119-1132

41. Heneka MT et al (2015) Neuroinflammation in Alzheimer's disease. Lancet Neurol 14:388-405

42. Rapic $\mathrm{S}$ et al (2013) Imaging microglial activation and glucose consumption in a mouse model of Alzheimer's disease. Neurobiol Aging 34:351-354

43. Yamagishi $\mathrm{S}$ et al (2019) Upregulation of cannabinoid receptor type 2, but not TSPO, in senescence-accelerated neuroinflammation in mice: a positron emission tomography study. J Neuroinflamm 16:208

44. Di Marzo V, Stella N, Zimmer A (2015) Endocannabinoid signalling and the deteriorating brain. Nat Rev Neurosci 16:30-42

45. Van Sickle MD et al (2005) Identification and functional characterization of brainstem cannabinoid CB2 receptors. Science 310:329-332

46. Xi ZX et al (2011) Brain cannabinoid $\mathrm{CB}(2)$ receptors modulate cocaine's actions in mice. Nat Neurosci 14:1160-1166

47. Benito C, Tolón RM, Pazos MR (2008) Cannabinoid CB2 receptors in human brain inflammation. Br J Pharmacol 153

48. Koppel J, et al (2014) CB2 receptor deficiency increases amyloid pathology and alters Tau processing in a transgenic mouse model of Alzheimer's disease. 20

49. Aso E, Ferrer I (2016) CB2 cannabinoid receptor as potential target against Alzheimer's disease. Front Neurosci 10

50. Parbo $\mathrm{P}$ et al (2017) Brain inflammation accompanies amyloid in the majority of mild cognitive impairment cases due to Alzheimer's disease. Brain 140:2002-2011

51. Spinelli F, Capparelli E, Abate C, Colabufo NA, Contino M (2017) Perspectives of cannabinoid type 2 receptor (CB2R) ligands in neurodegenerative disorders: structure-affinity relationship (SAfiR) and structure-activity relationship (SAR) studies. J Med Chem

52. Ni R, Mu L, Ametamey S (2019) Positron emission tomography of type 2 cannabinoid receptors for detecting inflammation in the central nervous system. Acta Pharmacol Sin 40:351-357

53. Spinelli F, Mu L, Ametamey SM (2018) Radioligands for positron emission tomography imaging of cannabinoid type 2 receptor. J Labelled Comp Radiopharm 61:299-308

54. Hou L et al (2021) Positron emission tomography imaging of the endocannabinoid system: opportunities and challenges in radiotracer development. J Med Chem 64:123-149

55. Varlow C, Boileau I, Wey HY, Liang SH, Vasdev N (2020) Classics in neuroimaging: imaging the endocannabinoid pathway with PET. ACS Chem Neurosci 11:1855-1862

56. Pottier $\mathrm{G}$ et al (2017) PET imaging of cannabinoid type 2 receptors with [11C]A-836339 did not evidence changes following neuroinflammation in rats. J Cereb Blood Flow Metab 37:1163-1178

57. Moldovan R-P et al (2015) 18F-JHU94620, a high affinity PET radioligand for imaging of cannabinoid subtype 2 receptors (CB2R). J Nucl Med 56:1048-1048

58. Attili B et al (2019) Preclinical evaluation of [(18) F]MA3: a CB2 receptor agonist radiotracer for PET. Br J Pharmacol 176:1481-1491

59. Heimann D et al (2018) Optimization of pharmacokinetic properties by modification of a carbazole-based cannabinoid receptor subtype 2 (CB(2)) ligand. Eur J Med Chem 143:1436-1447

60. Kallinen A et al (2019) Synthesis and in vitro evaluation of fluorine-18 benzimidazole sulfones as CB2 PET-radioligands. Org Biomol Chem 17:5086-5098

61. Haider A et al (2016) Synthesis and biological evaluation of thiophenebased cannabinoid receptor type 2 radiotracers for PET imaging. Front Neurosci 10:350

62. CaillÃ F et al (2017) Biodistribution of [18F] FC0324, a new PET tracer targeting CB2 receptors. J Nucl Med 58:855-855 
63. Haider A et al (2020) Identification and preclinical development of a 2,5,6-trisubstituted fluorinated pyridine derivative as a radioligand for the positron emission tomography imaging of cannabinoid type 2 receptors. J Med Chem 63:10287-10306

64. Offner $\mathrm{H}$ et al (2006) Experimental stroke induces massive, rapid activation of the peripheral immune system. J Cereb Blood Flow Metab 26:654-665

65. Garbuzova-Davis S et al (2016) Blood-spinal cord barrier alterations in subacute and chronic stages of a rat model of focal cerebral ischemia. J Neuropathol Exp Neurol 75:673-688

66. Huang B et al (2016) GlyT1 Inhibitor NFPS Exerts Neuroprotection via GlyR alpha1 subunit in the rat Model of transient focal cerebral ischaemia and reperfusion. Cell Physiol Biochem 38:1952-1962

67. Dang $\mathrm{G}$ et al (2016) Dynamic secondary degeneration in the spinal cord and ventral root after a focal cerebral infarction among hypertensive rats. Sci Rep 6:22655

68. Moisse K, Welch I, Hill T, Volkening K, Strong MJ (2008) Transient middle cerebral artery occlusion induces microglial priming in the lumbar spinal cord: a novel model of neuroinflammation. J Neuroinflamm $5: 29$

69. Dang $\mathrm{G}$ et al (2018) Alterations in the spinal cord and ventral root after cerebral infarction in non-human primates. Restor Neurol Neurosci 36:729-740

70. Saleh A et al (2004) In vivo MRI of brain inflammation in human ischaemic stroke. Brain 127:1670-1677

71. Love C, Tomas MB, Tronco GG, Palestro CJ (2005) FDG PET of infection and inflammation. Radiographics 25:1357-1368

72. Probasco JC, et al (2017) Abnormal brain metabolism on FDG-PET/CT is a common early finding in autoimmune encephalitis. Neurology $(R)$ $4: \mathrm{e} 352$

73. Ni R, Vaas M, Ren W, Klohs J (2018) Non-invasive detection of acute cerebral hypoxia and subsequent matrix-metalloproteinase activity in a mouse model of cerebral ischemia using multispectral-optoacoustictomography. Neurophotonics 5:015005

74. Vaas M, Ni R, Rudin M, Kipar A, Klohs J (2017) Extracerebral tissue damage in the intraluminal filament mouse model of middle cerebral artery occlusion. Front Neurol 8:85

75. Livak KJ, Schmittgen TD (2001) Analysis of relative gene expression data using real-time quantitative PCR and the 2(-delta delta C(T)) method. Methods 25:402-408

76. Ni R et al (2019) fMRI reveals mitigation of cerebrovascular dysfunction by bradykinin receptors 1 and 2 inhibitor noscapine in a mouse model of cerebral amyloidosis. Front Aging Neurosci 11:27-27

77. Ni R, Rudin M, Klohs J (2018) Cortical hypoperfusion and reduced cerebral metabolic rate of oxygen in the arcAbeta mouse model of Alzheimer's disease. Photoacoustics 10:38-47

78. Ren W, et al (2019) Automated registration of magnetic resonance imaging and optoacoustic tomography data for experimental studies. Neurophotonics 6:1-10

79. Vaas M et al (2018) Vascular and tissue changes of magnetic susceptibility in the mouse brain after transient cerebral ischemia. Transl Stroke Res 9:426-435

80. Krämer SD et al (2018) Evaluation of (11)C-Me-NB1 as a potential PET radioligand for measuring GluN2B-containing NMDA receptors, drug occupancy, and receptor cross talk. J Nucl Med 59:698-703
81. Lin TN, He YY, Wu G, Khan M, Hsu CY (1993) Effect of brain edema on infarct volume in a focal cerebral ischemia model in rats. Stroke 24:117-121

82. Ma Y et al (2005) A three-dimensional digital atlas database of the adult C57BL/6J mouse brain by magnetic resonance microscopy. Neuroscience 135:1203-1215

83. Pettigrew LC et al (1996) Microtubular proteolysis in focal cerebral ischemia. J Cereb Blood Flow Metab 16:1189-1202

84. Dawson DA, Hallenbeck JM (1996) Acute focal ischemia-induced alterations in MAP2 immunostaining: description of temporal changes and utilization as a marker for volumetric assessment of acute brain injury. J Cereb Blood Flow Metab 16:170-174

85. Hosoya $\mathrm{T}$ et al (2017) In vivo TSPO and cannabinoid receptor type 2 availability early in post-stroke neuroinflammation in rats: a positron emission tomography study. J Neuroinflamm 14:69

86. Vandeputte $C$ et al (2012) Small-animal PET imaging of the type 1 and type 2 cannabinoid receptors in a photothrombotic stroke model. Eur J Nucl Med Mol Imag 39:1796-1806

87. Jordan CJ, Xi Z-X (2019) Progress in brain cannabinoid CB(2) receptor research: from genes to behavior. Neurosci Biobehav Rev 98:208-220

88. Fernández-López D et al (2012) Reduced infarct size and accumulation of microglia in rats treated with WIN 55,212-2 after neonatal stroke. Neuroscience 207:307-315

89. Zhang $\mathrm{M}$ et al (2008) Modulation of the balance between cannabinoid $\mathrm{CB}(1)$ and $\mathrm{CB}(2)$ receptor activation during cerebral ischemic/reperfusion injury. Neuroscience 152:753-760

90. Govaerts SJ, Hermans E, Lambert DM (2004) Comparison of cannabinoid ligands affinities and efficacies in murine tissues and in transfected cells expressing human recombinant cannabinoid receptors. Eur J Pharm Sci 23:233-243

91. Yuan H et al (2013) Spatiotemporal uptake characteristics of [18] F-2-fluoro-2-deoxy-d-glucose in a Rat middle cerebral artery occlusion model. Stroke 44:2292-2299

92. Walberer $\mathrm{M}$ et al (2012) Potential of early [(18)F]-2-fluoro-2-deoxyD-glucose positron emission tomography for identifying hypoperfusion and predicting fate of tissue in a rat embolic stroke model. Stroke 43:193-198

93. Jin T, Mehrens H, Wang P, Kim S-G (2017) Chemical exchangesensitive spin-lock MRI of glucose analog 3-O-methyl- d -glucose in normal and ischemic brain. J Cerebral Blood Flow Metab 38: $0271678 X 1770741$

94. Liu N-W, et al (2017) Evolutional characterization of photochemically induced stroke in rats: a multimodality imaging and molecular biological study. Transl Stroke Res 8

95. Backes $\mathrm{H}$ et al (2016) Glucose consumption of inflammatory cells masks metabolic deficits in the brain. Neuroimage 128:54-62

96. Sobrado $\mathrm{M}$ et al (2011) Longitudinal studies of ischemic penumbra by using 18F-FDG PET and MRI techniques in permanent and transient focal cerebral ischemia in rats. Neuroimage 57:45-54

Publisher's Note Springer Nature remains neutral with regard to jurisdictional claims in published maps and institutional affiliations. 